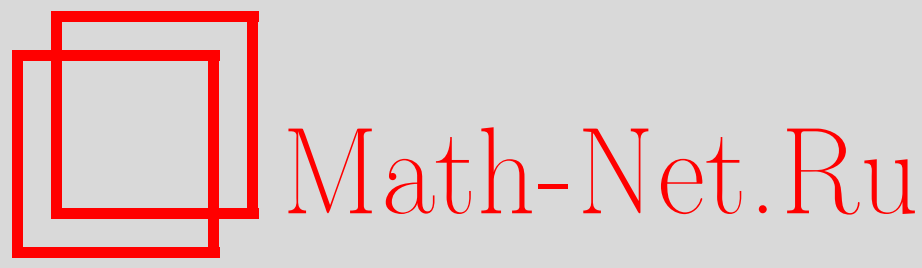

В. А. Фатеев, А. В. Литвинов, Многоточечные корреляционные функции в лиувиллевской теории поля и минимальной лиувиллевской гравитации, ТМФ, 2008, том 154, номер 3, 536-556

DOI: https://doi.org/10.4213/tmf6185

Использование Общероссийского математического портала Math-Net.Ru подразумевает, что вы прочитали и согласны с пользовательским соглашением http://www.mathnet.ru/rus/agreement

Параметры загрузки:

IP : 54.164 .48 .24

26 апреля 2023 г., 13:12:14

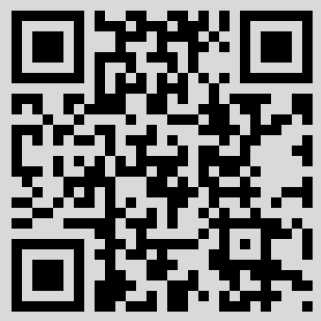




\section{МНОГОТОЧЕЧНЫЕ КОРРЕЛЯЦИОННЫЕ ФУНКЦИИ В ЛИУВИЛЛЕВСКОЙ ТЕОРИИ ПОЛЯ И МИНИМАЛЬНОЙ ЛИУВИЛЛЕВСКОЙ ГРАВИТАЦИИ}

Исследуются $(n+3)$-точечные корреляционные функции экспоненциальных полей в лиувиллевской теории поля с $n$ вырожденными и тремя произвольными полями. Аналитическое выражение для этих корреляционных функций выведено в терминах кулоновских интегралов. Рассмотрено применение этих результатов к минимальной лиувиллевской гравитации.

Ключевые слова: конформная теория поля, интегрируемые модели, теория Лиувилля, некритическая теория струн.

\section{1. ЛИУВИЛЛЕВСКАЯ ТЕОРИЯ ПОЛЯ}

В последнее время после основополагающей статьи Полякова [1] лиувиллевская теория поля (ЛТП) привлекает значительное внимание главным образом из-за ее глубоких связей с теорией бозонных струн в некритической размерности. ЛТП дает пример нерациональной конформной теории поля, которая имеет различные физические применения. Некоторые результаты, полученные в ЛТП и минимальной лиувиллевской гравитации, можно сравнить с результатами матричных моделей [2]-[8]. Для специального набора полей (так называемых вырожденных полей) корреляционные функции в ЛТП просто связаны с корреляционными функциями в минимальных моделях конформной теории поля, которые описывают критическое поведение многих интересных двумерных статистических систем.

Лиувиллевская теория поля описывается локальной плотностью лагранжиана

$$
\mathcal{L}=\frac{1}{4 \pi}\left(\partial_{a} \varphi\right)^{2}+\mu e^{2 b \varphi}
$$

с голоморфным тензором энергии-импульса

$$
T(z)=-\left(\partial_{z} \varphi\right)^{2}+Q \partial_{z}^{2} \varphi
$$

${ }^{*}$ Институт теоретической физики им. Л. Д. Ландау, Черноголовка, Московская обл., Россия. E-mail: litvinov@itp.ac.ru

${ }^{\dagger}$ Laboratoire de Physique Théorique et Astroparticules, Université Montpellier 2, Montpellier, France. E-mail: Vladimir.FATEEV@LPTA.univ-montp2.fr 
который обеспечивает локальную конформную инвариантность теории с центральным зарядом $c_{\mathrm{L}}$, параметризованным в терминах константы взаимодействия $b$ как $c_{\mathrm{L}}=1+6 Q^{2}$, где $Q=b+b^{-1}$. Масштабный параметр $\mu$ в (1.1) называется космологической постоянной.

Основными объектами этой теории являются экспоненциальные поля, параметризованные непрерывным параметром $\alpha$,

$$
V_{\alpha}(z, \bar{z})=e^{2 \alpha \varphi} \text {. }
$$

Они являются примарными полями алгебры Вирасоро, порожденной тензором энергии-импульса $T(z)$, с конформной размерностью $\Delta_{\mathrm{L}}(\alpha)=\alpha(Q-\alpha)$. Здесь и далее $z$ - комплексная координата на плоскости: $z=x_{1}+i x_{2}$. Для упрощения обозначений будем записывать примарное поле, определенное равенством $(1.2)$, как $V_{\alpha}(z)$ и положим $d^{2} z=d x_{1} d x_{2}$. Важное свойство ЛТП состоит в том, что поля $V_{\alpha}$ и $V_{Q-\alpha}$ имеют одну и ту же конформную размерность и на самом деле представляют одно и то же конформное поле. Это означает, что они связаны линейным преобразованием

$$
V_{\alpha}=R(\alpha) V_{Q-\alpha}
$$

с функцией

$$
R(\alpha)=\frac{\left(\pi \mu \gamma\left(b^{2}\right)\right)^{(Q-2 \alpha) / b}}{b^{2}} \frac{\gamma\left(2 b \alpha-b^{2}\right)}{\gamma\left(2-2 \alpha / b+1 / b^{2}\right)},
$$

известной как амплитуда отражения. Здесь и далее мы используем обозначение $\gamma(x)=\Gamma(x) / \Gamma(1-x)$.

В настоящей работе мы рассматриваем корреляционные функции в ЛТП, содержащие три произвольных и $n$ вырожденных полей. Вырожденные поля в ЛТП соответствуют примарным полям $V_{\alpha}(z)$ со значениями параметра $\alpha$, равными ${ }^{1)}$

$$
\alpha=-\frac{m b}{2}-\frac{n b^{-1}}{2}, \quad m, n=0,1,2, \ldots
$$

Чтобы сделать все формулы в работе более простыми, мы рассмотрим только случай $n=0$. Корреляционные функции при $n \neq 0$ имеют более сложный вид, и мы предполагаем рассмотреть их в другой публикации.

Хорошо известно, что четырехточечная корреляционная функция с вырожденным полем $V_{-m b / 2}(z)$ в ЛТП удовлетворяет дифференциальному уравнению порядка $m+1$ по каждой из переменных $z$ и $\bar{z}$ [9]. Явный вид этой функции определяет интегральное представление для решений такого дифференциального уравнения. Корреляционные функции с тремя произвольными полями и более чем с одним вырожденным полем удовлетворяют уже системе дифференциальных уравнений в частных производных, и возможность записать решение этой системы в терминах конечномерных интегралов не очевидна. Решение конформной бутстрапной задачи для этих корреляционных функций в терминах конечномерных кулоновских интегралов является основным результатом данной работы. В случае, когда более трех

1) Равенство (1.4) следует понимать с точностью до преобразования (1.3). 
полей являются невырожденными, корреляционная функция содержит бесконечное число конформных блоков и нет оснований ожидать, что ее удастся записать в виде конечномерного интеграла. В данной работе мы выразим многоточечные корреляционные функции через интегралы по всей плоскости. В области сходимости эти интегралы полностью определяют корреляционные функции. Вне этой области их надо понимать в смысле аналитического продолжения. Это продолжение можно выполнить, например, переписывая интегралы по плоскости в терминах контурных интегралов, как это описано в работах [10], [11] (см. также приложение А).

Корреляционные функции в ЛТП с тремя произвольными и $m$ вырожденными полями имеют много различных применений. В ЛТП (или в минимальных моделях конформной теории поля), возмущенной вырожденным полем, они позволяют выразить пертурбативные поправки к трехточечным корреляционным функциям (структурным константам операторного произведения) в терминах конечномерных интегралов. Даже в минимальных моделях конформной теории поля [9], где для всех ненулевых корреляционных функций выполнено условие экранирования, наши результаты дают независимое интегральное представление (которое во многих случаях проще), число интегрирований в котором не зависит от трех произвольных полей. Эти многоточечные корреляционные функции также появляются и играют важную роль при изучении конформной теории поля Тоды. Мы предполагаем обсудить приложения многоточечных корреляционных функций в ЛТП к возмущенным конформным теориям поля и конформным теориям поля Тоды в других публикациях. В разделе 2 мы кратко рассмотрим применение этих функций к минимальной лиувиллевской гравитации.

Многоточечные корреляционные функции в ЛТП являются довольно сложными объектами, однако, как было замечено в работе [12], любая многоточечная корреляционная функция $\left\langle V_{\alpha_{1}}\left(z_{1}\right) \ldots V_{\alpha_{m}}\left(z_{m}\right)\right\rangle$ имеет полюс по переменной

$$
\alpha=\sum_{k=1}^{m} \alpha_{k},
$$

если выполнено условие экранирования $\alpha=Q-n b$, причем вычет выражается в терминах $2 n$-мерного кулоновского интеграла ${ }^{2)}$. А именно,

$$
\begin{aligned}
\left.\operatorname{res}\right|_{\alpha}=Q-n b & \left\langle V_{\alpha_{1}}\left(z_{1}\right) \ldots V_{\alpha_{m}}\left(z_{m}\right)\right\rangle= \\
& =(-\pi \mu)^{n} \prod_{i<j}\left|z_{i}-z_{j}\right|^{-4 \alpha_{i} \alpha_{j}} \int \prod_{j=1}^{n} \prod_{k=1}^{m}\left|t_{j}-z_{k}\right|^{-4 b \alpha_{k}} \mathcal{D}_{n}^{-2 b^{2}}(t) d^{2} \vec{t}_{n},
\end{aligned}
$$

где $\mathcal{D}_{n}(t)$ - квадрат модуля определителя Вандермонда

$$
\mathcal{D}_{n}(t)=\prod_{i<j}^{n}\left|t_{i}-t_{j}\right|^{2} .
$$

\footnotetext{
${ }^{2)}$ Подынтегральное выражение в (1.5) представляет ожидаемое значение полей $V_{\alpha_{k}}$ с $n$ экранирующими полями $V_{b}\left(t_{i}\right)$ над вакуумным состоянием безмассового свободного поля.
} 
Далее мы используем обозначение для меры

$$
d^{2} \vec{t}_{n}=\frac{1}{\pi^{n} n !} \prod_{k=1}^{n} d^{2} t_{k}
$$

В случае трех точек интеграл в правой части (1.5) имеет вид ${ }^{3)}$

$$
I_{n}\left(\alpha_{1}, \alpha_{2}, \alpha_{3}\right)=\int \prod_{k=1}^{n}\left|t_{k}\right|^{-4 b \alpha_{1}}\left|t_{k}-1\right|^{-4 b \alpha_{2}} \mathcal{D}_{n}^{-2 b^{2}}(t) d^{2} \vec{t}_{n} .
$$

Интеграл в (1.6) был вычислен точно в работе [11]. Здесь мы дадим другой, более простой вывод, основанный на интегральном соотношении [13], которое будет использовано в дальнейшем:

$$
\begin{aligned}
\int \mathcal{D}_{n}(y) & \prod_{i=1}^{n} \prod_{j=1}^{n+m+1}\left|y_{i}-t_{j}\right|^{2 p_{j}} d^{2} \vec{y}_{n}= \\
= & \frac{\prod_{j} \gamma\left(1+p_{j}\right)}{\gamma\left(1+n+\sum_{j} p_{j}\right)} \prod_{i<j}\left|t_{i}-t_{j}\right|^{2+2 p_{i}+2 p_{j}} \times \\
& \times \int \mathcal{D}_{m}(u) \prod_{i=1}^{m} \prod_{j=1}^{n+m+1}\left|u_{i}-t_{j}\right|^{-2-2 p_{j}} d^{2} \vec{u}_{m}
\end{aligned}
$$

А именно, следует применить тождество (1.7) справа налево при $m=0$ и подставить

$$
\mathcal{D}_{n}^{-2 b^{2}}(t)=\mathcal{D}_{n}(t) \frac{\gamma\left(-n b^{2}\right)}{\gamma^{n}\left(-b^{2}\right)} \int \mathcal{D}_{n-1}(y) \prod_{i=1}^{n-1} \prod_{j=1}^{n}\left|y_{i}-t_{j}\right|^{-2-2 b^{2}} d^{2} \vec{y}_{n-1}
$$

в правую часть (1.6). После этого интеграл по переменным $t_{j}$ можно вычислить, используя (1.7). Оставшийся интеграл по $y_{i}$ имеет тот же тип, что и (1.6), но с меньшим числом интегрирований. В результате выводим функциональное соотношение

$$
\begin{aligned}
& I_{n}\left(\alpha_{1}, \alpha_{2}, \alpha_{3}\right)= \\
& \quad=\frac{\gamma\left(-n b^{2}\right)}{\gamma\left(-b^{2}\right)} \frac{1}{\gamma\left(2 b \alpha_{1}\right) \gamma\left(2 b \alpha_{2}\right) \gamma\left(2 b \alpha_{3}+(n-1) b^{2}\right)} I_{n-1}\left(\alpha_{1}+\frac{b}{2}, \alpha_{2}+\frac{b}{2}, \alpha_{3}\right) .
\end{aligned}
$$

Повторяя эту процедуру $n$ раз, получаем, что

$$
I_{n}\left(\alpha_{1}, \alpha_{2}, \alpha_{3}\right)=\prod_{k=1}^{n} \frac{\gamma\left(-k b^{2}\right)}{\gamma\left(-b^{2}\right)} \prod_{j=0}^{n-1} \frac{1}{\gamma\left(2 b \alpha_{1}+j b^{2}\right) \gamma\left(2 b \alpha_{2}+j b^{2}\right) \gamma\left(2 b \alpha_{3}+j b^{2}\right)}
$$

Функциональное соотношение (1.9) можно использовать для продолжения функции $I_{n}\left(\alpha_{1}, \alpha_{2}, \alpha_{3}\right)$ на нецелые $n$ (число экранирующих полей $\left.V_{b}\right)$. Подставим

$$
n=\frac{Q-\alpha_{1}-\alpha_{2}-\alpha_{3}}{b}
$$

\footnotetext{
${ }^{3)}$ Используя проективную инвариантность, можно положить в (1.5) $z_{1}=0, z_{2}=1$ и $z_{3}=\infty$.
} 
в равенство (1.9) и рассмотрим его как функциональное соотношение для трехточечной функции $C\left(\alpha_{1}, \alpha_{2}, \alpha_{3}\right)$, которая удовлетворяет условию

$$
\text { res }\left.\right|_{\alpha=Q-n b} C\left(\alpha_{1}, \alpha_{2}, \alpha_{3}\right) \stackrel{\text { def }}{=}(-\pi \mu)^{n} I_{n}\left(\alpha_{1}, \alpha_{2}, \alpha_{3}\right) \text {. }
$$

Функциональное соотношение (1.9), где $n$ определено в (1.10), вместе с условием (1.11) позволяет найти функцию $C\left(\alpha_{1}, \alpha_{2}, \alpha_{3}\right)$. Аналитическое выражение для $C\left(\alpha_{1}, \alpha_{2}, \alpha_{3}\right)$ было предложено в работах [14]-[16]:

$$
C\left(\alpha_{1}, \alpha_{2}, \alpha_{3}\right)=\left[\pi \mu \gamma\left(b^{2}\right) b^{2-2 b^{2}}\right]^{(Q-\alpha) / b} \frac{\Upsilon^{\prime}(0) \prod_{k=1}^{3} \Upsilon\left(2 \alpha_{k}\right)}{\Upsilon(\alpha-Q) \prod_{k=1}^{3} \Upsilon\left(\alpha-2 \alpha_{k}\right)},
$$

где $\Upsilon(x)$ - целая функция, самодуальная по отношению к $b \rightarrow b^{-1}$, удовлетворяющая функциональным соотношениям

$$
\Upsilon(x+b)=\gamma(b x) b^{1-2 b x} \Upsilon(x), \quad \Upsilon\left(x+b^{-1}\right)=\gamma\left(b^{-1} x\right) b^{2 b^{-1} x-1} \Upsilon(x)
$$

и определяемая интегралом

$$
\ln \Upsilon(x)=\int_{0}^{\infty} \frac{d t}{t}\left[\left(\frac{Q}{2}-x\right)^{2} e^{-t}-\frac{\operatorname{sh}^{2}(Q / 2-x)(t / 2)}{\operatorname{sh}(b t / 2) \operatorname{sh}(t /(2 b))}\right] .
$$

Эта функция симметрична, $\Upsilon(x)=\Upsilon(Q-x)$, и имеет нули в точках

$$
x=\left\{\begin{array}{l}
-m b-n b^{-1}, \\
Q+m b+n b^{-1},
\end{array} \quad m, n=0,1,2, \ldots .\right.
$$

Легко видеть, что функция $C\left(\alpha_{1}, \alpha_{2}, \alpha_{3}\right)$ удовлетворяет соотношению (1.9), где $n$ определяется условием (1.10).

В случае четырех точек корреляционная функция имеет значительно более сложную аналитическую структуру. Однако ситуация упрощается в случае, когда один из параметров $\alpha_{k}=-m b / 2$ при $m=0,1,2, \ldots$. Для этих значений параметров поле $V_{-m b / 2}(z)$ является вырожденным на уровне $m+1$, и четырехточечная корреляционная функция удовлетворяет дифференциальному уравнению одного и того же порядка по каждой переменной $z$ и $\bar{z}$. Это дает основания ожидать, что ее можно представить конечномерным интегралом. Чтобы найти это интегральное представление, начнем со случая, когда выполнено условие

$$
-\frac{m b}{2}+\alpha_{1}+\alpha_{2}+\alpha_{3}+n b=Q
$$

т.е. корреляционная функция обладает полюсом по переменной $\alpha=\alpha_{1}+\alpha_{2}+\alpha_{3}$ с вычетом

$$
\begin{aligned}
\left.\operatorname{res}\right|_{\alpha} & =Q-n b+m b / 2 \\
& =(-\pi \mu)^{n}|z|^{2 m b \alpha_{1}}|z-1|^{2 m b \alpha_{2}} J_{m}^{(n)}\left(\alpha_{1}, \alpha_{2}, \alpha_{3} \mid z\right),
\end{aligned}
$$


где

$$
J_{m}^{(n)}\left(\alpha_{1}, \alpha_{2}, \alpha_{3} \mid z\right)=\int \prod_{k=1}^{n}\left|t_{k}\right|^{-4 b \alpha_{1}}\left|t_{k}-1\right|^{-4 b \alpha_{2}}\left|t_{k}-z\right|^{2 m b^{2}} \mathcal{D}_{n}^{-2 b^{2}}(t) d^{2} \vec{t}_{n}
$$

a $n$ - число экранирующих полей $V_{b}$. Можно уменьшить число интегрирований в (1.14) путем многократного применения соотношения $(1.7)^{4)}$. А именно, можно показать [17], что интеграл (1.14) удовлетворяет следующему равенству:

$$
J_{m}^{(n)}\left(\alpha_{1}, \alpha_{2}, \alpha_{3} \mid z\right)=\Omega_{m}^{n}\left(\alpha_{1}, \alpha_{2}, \alpha_{3}\right) J_{n}^{(m)}\left(\tilde{\alpha}_{1}, \tilde{\alpha}_{2}, \tilde{\alpha}_{3} \mid z\right),
$$

где $\tilde{\alpha}_{k}=\alpha_{k}+(n-m) b / 2$,

$$
\begin{aligned}
& \Omega_{m}^{n}\left(\alpha_{1}, \alpha_{2}, \alpha_{3}\right)= \\
& \quad=\prod_{k=1}^{n-m} \frac{\gamma\left(-(k+m) b^{2}\right)}{\gamma\left(-b^{2}\right)} \prod_{j=0}^{n-m-1} \frac{1}{\gamma\left(2 b \alpha_{1}+j b^{2}\right) \gamma\left(2 b \alpha_{2}+j b^{2}\right) \gamma\left(2 b \alpha_{3}+j b^{2}\right)} .
\end{aligned}
$$

Далее, $n$ входит в интеграл $J_{n}^{(m)}\left(\tilde{\alpha}_{1}, \tilde{\alpha}_{2}, \tilde{\alpha}_{3} \mid z\right)$ как параметр и легко выполнить продолжение на нецелые $n$, связанные с $\alpha$ формулой (1.13). Продолжение функции $\Omega_{m}^{n}\left(\alpha_{1}, \alpha_{2}, \alpha_{3}\right)$ следует сделать так, чтобы

$$
\text { res }\left.\right|_{\alpha=Q-n b+m b / 2} \Omega_{m}\left(\alpha_{1}, \alpha_{2}, \alpha_{3}\right)=(-\pi \mu)^{n} \Omega_{m}^{n}\left(\alpha_{1}, \alpha_{2}, \alpha_{3}\right) .
$$

В итоге получаем следующее выражение для четырехточечной функции с одним вырожденным полем в ЛТП:

$$
\begin{gathered}
\left\langle V_{-m b / 2}(z) V_{\alpha_{1}}(0) V_{\alpha_{2}}(1) V_{\alpha_{3}}(\infty)\right\rangle= \\
=\Omega_{m}\left(\alpha_{1}, \alpha_{2}, \alpha_{3}\right)|z|^{2 m b \alpha_{1}}|z-1|^{2 m b \alpha_{2}} \mathbf{J}_{m}(A, B, C \mid z), \\
\mathbf{J}_{m}(A, B, C \mid z)=\int \prod_{k=1}^{m}\left|t_{k}\right|^{2 A}\left|t_{k}-1\right|^{2 B}\left|t_{k}-z\right|^{2 C} \mathcal{D}_{m}^{-2 b^{2}}(t) d^{2} \vec{t}_{m},
\end{gathered}
$$

где

$$
A=b\left(\alpha-2 \alpha_{1}-Q+\frac{m b}{2}\right), \quad B=b\left(\alpha-2 \alpha_{2}-Q+\frac{m b}{2}\right), \quad C=b\left(Q+\frac{m b}{2}-\alpha\right),
$$

а нормировочная постоянная $\Omega_{m}\left(\alpha_{1}, \alpha_{2}, \alpha_{3}\right)$ равна

$$
\begin{aligned}
\Omega_{m}\left(\alpha_{1}, \alpha_{2}, \alpha_{3}\right)=( & -\pi \mu)^{m}\left[\pi \mu \gamma\left(b^{2}\right) b^{2-2 b^{2}}\right]^{(Q-\alpha-m b / 2) / b} \times \\
& \times \frac{\Upsilon^{\prime}(-m b) \prod_{k=1}^{3} \Upsilon\left(2 \alpha_{k}\right)}{\Upsilon(\alpha-Q-m b / 2) \prod_{k=1}^{3} \Upsilon\left(\alpha-2 \alpha_{k}+m b / 2\right)},
\end{aligned}
$$

где $\alpha=\alpha_{1}+\alpha_{2}+\alpha_{3}$.

\footnotetext{
4) Мы временно предполагаем, что $n \geqslant m$.
} 
Можно обобщить результаты (1.12) и (1.16) на многоточечные корреляционные функции. Например, для пятиточечной корреляционной функции с двумя вырожденными полями $\left\langle V_{-\left(m_{1} b\right) / 2}\left(z_{1}\right) V_{-\left(m_{2} b\right) / 2}\left(z_{2}\right) V_{\alpha_{1}}(0) V_{\alpha_{2}}(1) V_{\alpha_{3}}(\infty)\right\rangle$ можно получить выражение

$$
\begin{aligned}
&\left\langle V_{-\left(m_{1} b\right) / 2}\left(z_{1}\right) V_{-\left(m_{2} b\right) / 2}\left(z_{2}\right) V_{\alpha_{1}}(0) V_{\alpha_{2}}(1) V_{\alpha_{3}}(\infty)\right\rangle== \\
&=\Omega_{m_{1} m_{2}}\left(\alpha_{1}, \alpha_{2}, \alpha_{3}\right)\left|z_{1}\right|^{2 m_{1} b \alpha_{1}}\left|z_{1}-1\right|^{2 m_{1} b \alpha_{2}} \times \\
& \quad \times\left|z_{2}\right|^{2 m_{2} b\left(Q-\alpha_{1}\right)}\left|z_{2}-1\right|^{2 m_{2} b\left(Q-\alpha_{2}\right)} \times \\
& \quad \times\left|z_{1}-z_{2}\right|^{-m_{1} m_{2} b^{2}} \mathbf{J}_{m_{1} m_{2}}\left(A, B, C \mid z_{1}, z_{2}\right),
\end{aligned}
$$

где

$$
\begin{aligned}
\mathbf{J}_{m_{1} m_{2}}\left(A, B, C \mid z_{1}, z_{2}\right)=\int & \mathcal{D}_{m_{1}}^{-2 b^{2}}(t) \mathcal{D}_{m_{2}}^{-2 b^{2}}(y) \prod_{j=1}^{m_{1}} \prod_{k=1}^{m_{2}}\left|t_{j}-y_{k}\right|^{2 b^{2}} \times \\
& \times \prod_{j=1}^{m_{1}}\left|t_{j}\right|^{2 A}\left|t_{j}-1\right|^{2 B}\left|t_{j}-z_{1}\right|^{2 C} d^{2} \vec{t}_{m_{1}} \times \\
& \times \prod_{k=1}^{m_{2}}\left|y_{k}\right|^{-2\left(1+b^{2}+A\right)}\left|y_{k}-1\right|^{-2\left(1+b^{2}+B\right)} \times \\
& \times\left|y_{k}-z_{2}\right|^{-2\left(1-\left(m_{1}+m_{2}-1\right) b^{2}+C\right)} d^{2} \vec{y}_{m_{2}}
\end{aligned}
$$

при

$$
\begin{gathered}
A=b\left(\alpha-2 \alpha_{1}-Q+\frac{\left(m_{1}-m_{2}\right) b}{2}\right), \quad B=b\left(\alpha-2 \alpha_{2}-Q+\frac{\left(m_{1}-m_{2}\right) b}{2}\right), \\
C=b\left(Q+\frac{\left(m_{1}+m_{2}\right) b}{2}-\alpha\right)
\end{gathered}
$$

а нормировочный множитель $\Omega_{m_{1} m_{2}}\left(\alpha_{1}, \alpha_{2}, \alpha_{3}\right)$ равен

$$
\begin{aligned}
& \Omega_{m_{1} m_{2}}\left(\alpha_{1}, \alpha_{2}, \alpha_{3}\right)=(-\pi \mu)^{m_{1}+m_{2}} \times \\
& \quad \times\left[\pi \mu \gamma\left(b^{2}\right) b^{2-2 b^{2}}\right]^{\left(Q-\alpha-\left(m_{1}+m_{2}\right) b / 2\right) / b} \frac{\Upsilon^{\prime}\left(-m_{1} b\right) \Upsilon^{\prime}\left(-m_{2} b\right)}{\Upsilon^{\prime}(0)} \times \\
& \quad \times \frac{\Upsilon\left(\alpha-Q+\frac{\left(m_{1}-m_{2}\right) b}{2}\right)}{\Upsilon\left(\alpha-Q+\frac{\left(m_{1}+m_{2}\right) b}{2}\right) \Upsilon\left(\alpha-Q-\frac{\left(m_{1}+m_{2}\right) b}{2}\right)} \prod_{k=1}^{3} \frac{\Upsilon\left(2 \alpha_{k}\right)}{\Upsilon\left(\alpha-2 \alpha_{k}+\frac{\left(m_{1}-m_{2}\right) b}{2}\right)} .
\end{aligned}
$$

Заметим, что интеграл для пятиточечной корреляционной функции (1.19) имеет скрытую $s l(3)$-структуру. А именно, кулоновское взаимодействие между точками $t_{i}$ и $y_{j}$ в первых трех членах в (1.19) можно описывать матрицей Картана алгебры Ли $s l(3)$. Интегралы с этим типом кулоновского взаимодействия появляются в конформной теории поля Тоды [18]. Схожая $s l(m+1)$-структура возникает в кулоновских интегралах для $(m+3)$-точечной корреляционной функции с тремя произвольными полями. 
В принципе возможно найти аналитическое выражение для любой $(k+3)$-точечной корреляционной функции с $k$ вырожденными и тремя произвольными полями. Для этого достаточно записать интегральное представление для корреляционной функции с $m$ полями $V_{-b / 2}$ :

$$
\left\langle V_{-b / 2}\left(z_{1}\right) \ldots V_{-b / 2}\left(z_{m}\right) V_{\alpha_{1}}(0) V_{\alpha_{2}}(1) V_{\alpha_{3}}(\infty)\right\rangle
$$

так как корреляционную функцию с произвольным числом вырожденных полей можно получить из этой функции, используя свойство слияния

$$
V_{-m_{1} b / 2}(z) V_{-m_{2} b / 2}(0)=|z|^{-m_{1} m_{2} b^{2}} V_{-\left(m_{1}+m_{2}\right) b / 2}(0)+\cdots .
$$

Явное выражение для корреляционной функции (1.20) можно записать в терминах вспомогательной функции (ядра) $K_{m}^{\Delta}\left(t_{1}, \ldots, t_{m} \mid y_{1}, \ldots, y_{m}\right)$, которая является симметричной функцией переменных $t_{k}$ и $y_{k}$ и не меняется при перестановке $t_{k} \leftrightarrow y_{k}$. Если исходить из явного вида функции $K_{m}^{\Delta}\left(t_{1}, \ldots, t_{m} \mid y_{1}, \ldots, y_{m}\right)$, то последние два свойства не очевидны, но их можно доказать. Эта функция имеет вид $m(m-1)$-мерного кулоновского интеграла, и представление для нее можно вывести из рекуррентных формул ${ }^{5)}$ :

$$
\begin{aligned}
& K_{m}^{\Delta}\left(t_{1}, \ldots, t_{m} \mid y_{1}, \ldots, y_{m}\right)=\frac{\gamma\left(-m b^{2}\right)}{\gamma^{m}\left(-b^{2}\right)} \mathcal{D}_{m}^{1+2 b^{2}}(t) \prod_{k=1}^{m}\left|t_{k}-y_{1}\right|^{2 \Delta} \times \\
& \quad \times \int \mathcal{D}_{m-1}(\tau) \prod_{j=1}^{m-1}\left|\tau_{j}-y_{1}\right|^{-2 \Delta+2 b^{2}} \prod_{k=1}^{m}\left|\tau_{j}-t_{k}\right|^{-2-2 b^{2}} \times \\
& \quad \times K_{m-1}^{\Delta+b^{2}}\left(\tau_{1}, \ldots, \tau_{m-1} \mid y_{2}, \ldots, y_{m}\right) d^{2} \vec{\tau}_{m-1}
\end{aligned}
$$

Мы даем вывод формулы (1.21) в случаях $m=1$ и $m=2$ и описываем общую процедуру для $m>2$ в приложении Б.

Являясь довольно сложной, эта функция имеет простую квазиклассическую асимптотику при $b \rightarrow 0$, если зафиксировать $\Delta$ :

$K_{m}^{\Delta}\left(t_{1}, \ldots, t_{m} \mid y_{1}, \ldots, y_{m}\right) \underset{b \rightarrow 0}{\longrightarrow} \frac{1}{m !}\left[\left|t_{1}-y_{1}\right|^{2 \Delta}\left|t_{2}-y_{2}\right|^{2 \Delta} \ldots\left|t_{m}-y_{m}\right|^{2 \Delta}+\right.$ symmetrization $]$.

Чтобы пояснить (1.21), сначала приведем три примера. Функция $K_{1}^{\Delta}(t \mid y)$ является простейшей, она имеет вид

$$
K_{1}^{\Delta}(t \mid y)=|t-y|^{2 \Delta} .
$$

Функция $K_{2}^{\Delta}\left(t_{1}, t_{2} \mid y_{1}, y_{2}\right)$ представляется в виде двумерного интеграла:

$$
\begin{aligned}
& K_{2}^{\Delta}\left(t_{1}, t_{2} \mid y_{1}, y_{2}\right)=\frac{\gamma\left(-2 b^{2}\right)}{\gamma^{2}\left(-b^{2}\right)}\left|t_{1}-t_{2}\right|^{2+4 b^{2}}\left|t_{1}-y_{1}\right|^{2 \Delta}\left|t_{2}-y_{1}\right|^{2 \Delta} \times \\
& \quad \times \int\left|\xi-y_{1}\right|^{-2 \Delta+2 b^{2}}\left|\xi-y_{2}\right|^{2 \Delta+2 b^{2}}\left|\xi-t_{1}\right|^{-2-2 b^{2}}\left|\xi-t_{2}\right|^{-2-2 b^{2}} d^{2} \vec{\xi}_{1} .
\end{aligned}
$$

5) Чтобы придать смысл этим формулам, мы положили $K_{0}^{\Delta}=1$. 
Свойства симметрии ядра (1.23) можно проверить, используя интегральное соотношение (А.2). Функция $K_{3}^{\Delta}\left(t_{1}, t_{2}, t_{3} \mid y_{1}, y_{2}, y_{3}\right)$ имеет вид шестимерного кулоновского интеграла:

$$
\begin{aligned}
& K_{3}^{\Delta}\left(t_{1}, t_{2}, t_{3} \mid y_{1}, y_{2}, y_{3}\right)=\frac{\gamma\left(-2 b^{2}\right) \gamma\left(-3 b^{2}\right)}{\gamma^{5}\left(-b^{2}\right)} \mathcal{D}_{3}^{1+2 b^{2}}(t) \prod_{k=1}^{3}\left|t_{k}-y_{1}\right|^{2 \Delta} \times \\
& \quad \times \int \mathcal{D}_{2}^{2+2 b^{2}}(\nu) \prod_{k=1}^{2}\left|\nu_{k}-y_{1}\right|^{-2 \Delta+2 b^{2}}\left|\nu_{k}-y_{2}\right|^{2 \Delta+2 b^{2}} \prod_{j=1}^{3}\left|\nu_{k}-t_{j}\right|^{-2-2 b^{2}} d^{2} \vec{\nu}_{2} \times \\
& \quad \times\left|\xi-y_{2}\right|^{-2 \Delta}\left|\xi-y_{3}\right|^{2 \Delta+4 b^{2}}\left|\xi-\nu_{1}\right|^{-2-2 b^{2}}\left|\xi-\nu_{2}\right|^{-2-2 b^{2}} d^{2} \vec{\xi}_{1},
\end{aligned}
$$

и т.д.

Корреляционную функцию (1.20) можно выразить в терминах ядра (1.21) следующим образом:

$$
\begin{aligned}
& \left\langle V_{-b / 2}\left(z_{1}\right) \ldots V_{-b / 2}\left(z_{m}\right) V_{\alpha_{1}}(0) V_{\alpha_{2}}(1) V_{\alpha_{3}}(\infty)\right\rangle= \\
& =\Omega_{m}\left(\alpha_{1}, \alpha_{2}, \alpha_{3}\right) \prod_{k=1}^{m}\left|z_{k}\right|^{2 b \alpha_{1}}\left|z_{k}-1\right|^{2 b \alpha_{2}} \times \\
& \quad \times \prod_{i<j}\left|z_{i}-z_{j}\right|^{-b^{2}} \int \prod_{k=1}^{m}\left|t_{k}\right|^{2 A}\left|t_{k}-1\right|^{2 B} \times \\
& \quad \times K_{m}^{C}\left(t_{1}, \ldots, t_{m} \mid z_{1}, \ldots, z_{m}\right) \mathcal{D}_{m}^{-2 b^{2}}(t) d^{2} \vec{t}_{m}
\end{aligned}
$$

где

$A=b\left(\alpha-2 \alpha_{1}-Q+\frac{m b}{2}\right), \quad B=b\left(\alpha-2 \alpha_{2}-Q+\frac{m b}{2}\right), \quad C=b\left(Q+\frac{(2-m) b}{2}-\alpha\right)$,

а нормировочный множитель $\Omega_{m}\left(\alpha_{1}, \alpha_{2}, \alpha_{3}\right)$ имеет вид (1.17).

Ядро (1.21), несмотря на его сложный вид, имеет много замечательных свойств, которые позволяют получать корреляционные функции для высших вырожденных полей $V_{-m b / 2}$. Например, оно имеет очень простую асимптотику при $y_{k} \rightarrow z$ :

$$
K_{m}^{\Delta}\left(t_{1}, \ldots, t_{m} \mid z, \ldots, z\right)=\prod_{k=1}^{m}\left|t_{k}-z\right|^{2 \Delta+2(m-1) b^{2}}
$$

Используя (1.25), из (1.24) можно получить представление (1.15) для четырехточечной функции. Другое важное свойство ядра (1.21) выражается равенством (А.3). Если рассмотреть $2 m$-мерный интеграл (1.24) при $m=m_{1}+m_{2}$ и "факторизовать" его, используя (А.3), на $2 m_{1}$ - и $2 m_{2}$-мерные интегралы, то, применяя (1.25), получаем выражение для пятиточечной корреляционной функции (1.18).

\section{2. МИНИМАЛЬНАЯ ЛИУВИЛЛЕВСКАЯ ГРАВИТАЦИЯ}

В данном разделе мы рассмотрим минимальную лиувиллевскую гравитацию, где материальная часть теории описывается обобщенной минимальной моделью (ОММ) 
конформной теории поля [4] с центральным зарядом $c_{\mathrm{M}}=1-6\left(b-b^{-1}\right)^{2}$, взаимодействующей с ЛТП таким образом, что $c_{\mathrm{L}}+c_{\mathrm{M}}=26$. ОММ включает континуальное число примарных полей $\Phi_{\alpha}$ с конформными размерностями $\Delta_{\mathrm{M}}(\alpha)=$ $\alpha\left(\alpha+b-b^{-1}\right)$. Многоточечные корреляционные функции этих полей обладают интегральными представлениями, если выполнено условие экранирования полей материи, т.е.

$$
\sum_{k=1}^{m} \alpha_{k}-n b=b^{-1}-b
$$

Тогда ${ }^{6)}$

$$
\begin{aligned}
\left\langle\Phi_{\alpha_{1}}\left(z_{1}\right) \ldots \Phi_{\alpha_{m}}\left(z_{m}\right)\right\rangle= & \frac{1}{N\left(b^{-1}-b\right)} \prod_{k=1}^{m} N\left(\alpha_{k}\right) \prod_{i<j}\left|z_{i}-z_{j}\right|^{4 \alpha_{i} \alpha_{j}} \times \\
& \times \int \prod_{j=1}^{n} \prod_{k=1}^{m}\left|t_{j}-z_{k}\right|^{-4 b \alpha_{k}} \mathcal{D}_{n}^{2 b^{2}}(t) d^{2} \vec{t}_{n}
\end{aligned}
$$

где нормировочный множитель

$$
N(\alpha)=\left(-\gamma\left(-b^{2}\right)\right)^{-\alpha / b}\left[\frac{\gamma\left(2 b^{-1} \alpha-b^{-2}+2\right)}{\gamma\left(2 b \alpha+b^{2}\right)} \frac{\gamma\left(b^{2}\right)}{\gamma\left(2-b^{-2}\right)}\right]^{1 / 2}
$$

выбран так, что

$$
\left\langle\Phi_{\alpha}(z) \Phi_{\alpha}\left(z^{\prime}\right)\right\rangle=\left|z-z^{\prime}\right|^{-4 \Delta_{\mathrm{M}}(\alpha)} .
$$

Одной из главных задач в минимальной лиувиллевской гравитации является вычисление корреляционных функций операторов $U_{\alpha}=\Phi_{\alpha-b} V_{\alpha}$, которые имеют конформную размерность $\Delta_{\mathrm{L}}(\alpha)+\Delta_{\mathrm{M}}(\alpha-b)=1$, а потому $(1,1)$-форму $U_{\alpha}(z) d^{2} z$ можно интегрировать инвариантным образом. Проинтегрированные $n$-точечные корреляционные функции являются инвариантными объектами, зависящими только от параметров $\alpha_{k}$. Из-за группы диффеоморфизмов $S L(2, C)$, которая является симметрией теории, число интегрирований в $(m+3)$-точечной корреляционной функции можно свести к $m$. А именно, можно фиксировать координаты любых трех полей в точках 0,1 и $\infty$. Таким образом, корреляционная функция (или, более правильно, корреляционное число) проинтегрированных операторов $U_{\alpha}$ можно определить как

$$
\begin{aligned}
\left\langle\left\langle U_{\alpha_{1}} \ldots U_{\alpha_{m+3}}\right\rangle\right\rangle \stackrel{\text { def }}{=} & \frac{1}{Z_{\mathrm{L}}} \int\left\langle V_{\alpha_{1}}\left(z_{1}\right) \ldots V_{\alpha_{m}}\left(z_{m}\right) V_{\alpha_{m+1}}(0) V_{\alpha_{m+2}}(1) V_{\alpha_{m+3}}(\infty)\right\rangle \times \\
& \times\left\langle\Phi_{\alpha_{1}-b}\left(z_{1}\right) \ldots \Phi_{\alpha_{m}-b}\left(z_{m}\right) \Phi_{\alpha_{m+1}-b}(0) \times\right. \\
& \left.\times \Phi_{\alpha_{m+2}-b}(1) \Phi_{\alpha_{m+3}-b}(\infty)\right\rangle d^{2} z_{1} \ldots d^{2} z_{m}
\end{aligned}
$$

где $Z_{\mathrm{L}}$ - лиувиллевская статсумма [4]

$$
Z_{\mathrm{L}}=\left(\pi \mu \gamma\left(b^{2}\right)\right)^{Q / b} \frac{1-b^{2}}{\pi^{3} \gamma\left(b^{2}\right) \gamma\left(b^{-2}\right) Q} .
$$

\footnotetext{
6) Заметим, что центральный заряд $c_{\mathrm{M}}$, конформную размерность $\Delta_{\mathrm{M}}(\alpha)$, условие экранирования (2.1) и корреляционную функцию (2.2) (с точностью до нормировки) можно вывести из соответствующих величин в ЛТП подстановкой $b \rightarrow-i b$ и $\alpha_{k} \rightarrow i \alpha_{k}$.
}

6 Теоретическая и математическая физика, т. 154, № 3, 2008 г. 
Трехточечная корреляционная функция полей $U_{\alpha_{k}}$ не содержит интегралов и имеет простой факторизованный вид [4]

$$
\left\langle\left\langle U_{\alpha_{1}} U_{\alpha_{2}} U_{\alpha_{3}}\right\rangle\right\rangle=b^{-2}\left(b^{-4}-1\right) \prod_{k=1}^{3} \mathcal{N}\left(\alpha_{k}\right),
$$

где мы ввели так называемые leg-множители [5], [6]

$$
\mathcal{N}(\alpha)=\pi\left(\pi \mu \gamma\left(b^{2}\right)\right)^{-\alpha / b}\left[\frac{\gamma\left(2 b \alpha-b^{2}\right) \gamma\left(2 b^{-1} \alpha-b^{-2}\right)}{\gamma\left(1-b^{2}\right) \gamma\left(2-b^{-2}\right)}\right]^{1 / 2}
$$

Четырехточечная функция (2.3) содержит одно интегрирование по z. Рассмотрим специальную четырехточечную функцию

$$
\left\langle\left\langle U_{-m b / 2} U_{\alpha_{1}} U_{\alpha_{2}} U_{\alpha_{3}}\right\rangle\right\rangle,
$$

которая содержит одно вырожденное лиувиллевское поле $V_{-m b / 2}$, а потому лиувиллевская часть корреляционной функции довольно проста и представима интегралом (1.16). Однако материальная часть этой корреляционной функции остается сложной. Чтобы упростить ее, предположим, что в материальном секторе выполнено условие экранирования

$$
-\frac{m b}{2}+\alpha_{1}+\alpha_{2}+\alpha_{3}-4 b-n b=b^{-1}-b .
$$

Тогда в силу (2.2) материальная часть корреляционной функции выражается через $2 n$-мерный кулоновский интеграл. При этих двух допущениях корреляционную функцию (2.5) можно выразить в терминах $2(n+m+1)$-мерного интеграла (с точностью до числовых множителей, которые возникают из (1.15) и (2.2)):

$$
\begin{aligned}
\mathbf{G}_{m}^{n}\left(\alpha_{1}, \alpha_{2}, \alpha_{3}\right)= & \mathcal{D}_{m}^{-2 b^{2}}(t) \mathcal{D}_{n}^{2 b^{2}}(\xi) \times \\
& \times \prod_{j=1}^{m}\left|t_{j}\right|^{2(m+n+2) b^{2}-4 b \alpha_{1}}\left|t_{j}-1\right|^{2(m+n+2) b^{2}-4 b \alpha_{2}}\left|t_{j}-z\right|^{-2(n+2) b^{2} \times} \\
& \times|z|^{2(m+2) b^{2}-4 b \alpha_{1}}|z-1|^{2(m+2) b^{2}-4 b \alpha_{2} \times} \\
& \times\left|\xi_{j}\right|^{-4 b\left(\alpha_{1}-b\right)}\left|\xi_{j}-1\right|^{-4 b\left(\alpha_{2}-b\right)}\left|\xi_{j}-z\right|^{2(m+2) b^{2}} d^{2} \vec{t}_{m} d^{2} \vec{\xi}_{n} d^{2} z .
\end{aligned}
$$

Интеграл (2.7) имеет очень простую структуру полюсов по каждой из переменных $\alpha_{i}$ и может быть вычислен точно (см. [17]):

$$
\begin{aligned}
\mathbf{G}_{m}^{n}\left(\alpha_{1}, \alpha_{2}, \alpha_{3}\right)= & \pi(m+1)(n+1) \prod_{j=1}^{m+1} \frac{\gamma\left(-j b^{2}\right)}{\gamma\left(-b^{2}\right)} \prod_{j=1}^{n+1} \frac{\gamma\left(j b^{2}\right)}{\gamma\left(b^{2}\right)} \times \\
& \times \prod_{j=2}^{m+n+2}\left[\gamma\left(2 b \alpha_{1}-j b^{2}\right) \gamma\left(2 b \alpha_{2}-j b^{2}\right) \gamma\left(2 b \alpha_{3}-j b^{2}\right)\right]^{-1}
\end{aligned}
$$


Собирая все нормировочные множители, полученные из равенств (1.15) и (2.2), приходим к окончательному простому результату:

$$
\left\langle\left\langle U_{-m b / 2} U_{\alpha_{1}} U_{\alpha_{2}} U_{\alpha_{3}}\right\rangle\right\rangle=b^{-2}\left(b^{-4}-1\right) \prod_{k=1}^{4} \mathcal{N}\left(\alpha_{k}\right)(m+1)(n+1),
$$

где $\alpha_{4}=-m b / 2$. Формула (2.8) верна в области сходимости интеграла (2.7):

$$
Q+b<\sum_{k=1}^{4} \alpha_{k}<2 Q-m b, \quad \frac{(m+1) b}{2}<\alpha_{j}<\frac{Q}{2}, \quad j=1,2,3 .
$$

В этой области можно выразить число экранирующих полей $n$ из равенства (2.6).

Пятиточечная функция (2.3) имеет два интегрирования по переменным $z_{1}$ и $z_{2}$. Рассмотрим специальную корреляционную функцию, которая содержит два вырожденных лиувиллевских поля

$$
\left\langle\left\langle U_{-m_{1} b / 2} U_{-m_{2} b / 2} U_{\alpha_{1}} U_{\alpha_{2}} U_{\alpha_{3}}\right\rangle\right\rangle
$$

и снова предположим, что в материальном секторе имеется условие экранирования

$$
-\frac{m_{1} b}{2}-\frac{m_{2} b}{2}+\alpha_{1}+\alpha_{2}+\alpha_{3}-5 b-n b=b^{-1}-b .
$$

Тогда корреляционную функцию (2.9) можно выразить в терминах $2\left(m_{1}+m_{2}+n+\right.$ $2)$-мерного интеграла (с точностью до числовых множителей, которые мы получили из (1.18) и (2.2)):

$$
\begin{aligned}
& \mathbf{G}_{m_{1} m_{2}}^{n}\left(\alpha_{1}, \alpha_{2}, \alpha_{3}\right)=\int \mathbf{J}_{m_{1} m_{2}}\left(A, B, C \mid z_{1}, z_{2}\right)\left|z_{1}\right|^{2\left(m_{1}+2\right) b^{2}-4 b \alpha_{1}}\left|z_{1}-1\right|^{2\left(m_{1}+2\right) b^{2}-4 b \alpha_{2} \times} \\
& \quad \times\left|z_{2}\right|^{-2+2\left(m_{2}+1\right)\left(1+2 b^{2}-2 b \alpha_{1}\right)}\left|z_{2}-1\right|^{-2+2\left(m_{2}+1\right)\left(1+2 b^{2}-2 b \alpha_{2}\right)}\left|z_{1}-z_{2}\right|^{2\left(m_{1}+m_{2}+2\right) b^{2}} \times \\
& \quad \times \prod_{j=1}^{n}\left|\xi_{j}\right|^{-4 b\left(\alpha_{1}-b\right)}\left|\xi_{j}-1\right|^{-4 b\left(\alpha_{2}-b\right)}\left|\xi_{j}-z_{1}\right|^{2\left(m_{1}+2\right) b^{2}} \times \\
& \quad \times\left|\xi_{j}-z_{2}\right|^{2\left(m_{2}+2\right) b^{2}} \mathcal{D}_{n}^{2 b^{2}}(\xi) d^{2} \vec{\xi}_{n} d^{2} z_{1} d^{2} z_{2},
\end{aligned}
$$

где функция $\mathbf{J}_{m_{1} m_{2}}\left(A, B, C \mid z_{1}, z_{2}\right)$ задается равенством (1.19) при

$$
A=\left(m_{1}+n+3\right) b^{2}-2 b \alpha_{1}, \quad B=\left(m_{1}+n+3\right) b^{2}-2 b \alpha_{2}, \quad C=-(n+3) b^{2} .
$$

Этот интеграл как функция параметров $\alpha_{i}$ также имеет простую структуру полюсов и может быть вычислен точно:

$$
\begin{aligned}
\mathbf{G}_{m_{1} m_{2}}^{n}\left(\alpha_{1}, \alpha_{2}, \alpha_{3}\right)= & \prod_{j=1}^{m_{1}+1} \frac{\gamma\left(-j b^{2}\right)}{\gamma\left(-b^{2}\right)} \prod_{j=1}^{m_{2}+1} \frac{\gamma\left(-j b^{2}\right)}{\gamma\left(-b^{2}\right)} \prod_{j=1}^{n+2} \frac{\gamma\left(j b^{2}\right)}{\gamma\left(b^{2}\right)} \times \\
& \times \prod_{j=1}^{m_{2}} \gamma\left(\left(m_{1}+n+2+j\right) b^{2}\right) \times \\
& \times \prod_{j=2}^{m_{1}+n+3}\left[\gamma\left(2 b \alpha_{1}-j b^{2}\right) \gamma\left(2 b \alpha_{2}-j b^{2}\right) \gamma\left(2 b \alpha_{3}-j b^{2}\right)\right]^{-1} .
\end{aligned}
$$


Собирая все необходимые множители, получаем простой результат для пятиточечной корреляционной функции

$$
\begin{aligned}
& \left\langle\left\langle U_{-m_{1} b / 2} U_{-m_{2} b / 2} U_{\alpha_{1}} U_{\alpha_{2}} U_{\alpha_{3}}\right\rangle\right\rangle= \\
& =b^{-2}\left(b^{-4}-1\right) \prod_{k=1}^{5} \mathcal{N}\left(\alpha_{k}\right)\left(m_{1}+1\right)\left(m_{2}+1\right)(n+1)(n+2),
\end{aligned}
$$

где $\alpha_{4}=-m_{1} b / 2$ и $\alpha_{5}=-m_{2} b / 2$. Представление (2.12) справедливо в области сходимости интеграла (2.11):

$$
Q+2 b<\sum_{k=1}^{5} \alpha_{k}<2 Q-\left(m_{1}+m_{2}\right) b, \quad \frac{\left(m_{1}+m_{2}+1\right) b}{2}<\alpha_{j}<\frac{Q}{2}, \quad j=1,2,3
$$

Равенства $(2.4),(2.8)$ и $(2.12)$ легко обобщить. Рассмотрим $(k+3)$-точечную корреляционную функцию с $k$ вырожденными лиувиллевскими полями

$$
\left\langle\left\langle U_{-m_{1} b / 2} \ldots U_{-m_{k} b / 2} U_{\alpha_{1}} U_{\alpha_{2}} U_{\alpha_{3}}\right\rangle\right\rangle
$$

и предположим, что в материальном секторе имеется условие экранирования (ср. с равенствами (2.6) и $(2.10))$

$$
-\frac{m_{1} b}{2}-\cdots-\frac{m_{k} b}{2}+\alpha_{1}+\alpha_{2}+\alpha_{3}-(k+3) b-n b=b^{-1}-b .
$$

Можно ожидать, что существует область в пространстве параметров $\alpha_{k}$, в которой соотношения $(2.4),(2.8)$ и $(2.12)$ допускают непосредственное обобщение

$$
\left\langle\left\langle U_{-m_{1} b / 2} \ldots U_{-m_{k} b / 2} U_{\alpha_{1}} U_{\alpha_{2}} U_{\alpha_{3}}\right\rangle\right\rangle=b^{-2}\left(b^{-4}-1\right) \prod_{j=1}^{k+3} \mathcal{N}\left(\alpha_{j}\right) \prod_{j=1}^{k}\left(m_{j}+1\right)(n+j),
$$

где $\alpha_{j+3}=-m_{j} b / 2$.

Все результаты данной работы можно переписать в “двойственном" случае, когда рассматривается $(k+3)$-точечная корреляционная функция с $k$ вырожденными материальными полями $\Phi_{m_{j} b / 2}$, т.е. коррелятор

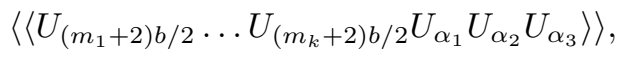

и предполагается, что в лиувиллевском секторе выполнено условие экранирования

$$
\frac{m_{1} b}{2}+\cdots+\frac{m_{k} b}{2}+\alpha_{1}+\alpha_{2}+\alpha_{3}+k b+n b=b+b^{-1},
$$

т.е. лиувиллевская часть корреляционной функции (2.13) имеет вид $2 n$-мерного интеграла в силу (1.5). В этом случае материальную часть корреляционной функции (2.13)

$$
\left\langle\Phi_{m_{1} b / 2} \ldots \Phi_{m_{k} b / 2} \Phi_{\alpha_{1}-b} \Phi_{\alpha_{2}-b} \Phi_{\alpha_{3}-b}\right\rangle
$$


можно представить интегралами, подобными полученным в разделе 1 для ЛТП. А именно, интегралы для $(k+3)$-точечной корреляционной функции в OMM с $k$ вырожденными полями $\Phi_{m_{j} b / 2}$ и тремя произвольными полями $\Phi_{\alpha_{j}}$ можно получить заменой $\alpha_{j} \rightarrow i \alpha_{j}$ и $b \rightarrow-i b$ в интегралах для $(k+3)$-точечной функции в ЛТП с $k$ вырожденными и тремя произвольными полями $V_{\alpha_{j}}$ (заметим, что в корреляционной функции (2.14) параметры $\alpha_{j}$ сдвинуты: $\left.\alpha_{j} \rightarrow \alpha_{j}-b\right)$. Однако общий численный множитель дается другим выражением. Этот множитель в случае четырехточечной корреляционной функции можно найти в работе [17], где рассмотрена четырехточечная корреляционная функция (2.13).

\section{ПРИЛОЖЕНИЕ А}

\section{Полезные интегральные тождества}

В этом приложении мы приведем без доказательства полезное интегральное соотношение, которое позволяет существенно уменьшить число интегрирований в корреляционной функции (1.24) и помогает упростить вычисление корреляционных функций.

Обобщение соотношения (1.7). Вместо соотношения (1.7) иногда удобнее использовать соотношение

$$
\begin{aligned}
& \int \mathcal{D}_{n}(y) \prod_{i=1}^{n} \prod_{j=1}^{n+m+2}\left|y_{i}-t_{j}\right|^{2 p_{j}} d^{2} \vec{y}_{n}=\prod_{j=1}^{n+m+2} \gamma\left(1+p_{j}\right) \prod_{i<j}\left|t_{i}-t_{j}\right|^{2+2 p_{i}+2 p_{j}} \times \\
& \quad \times \int \mathcal{D}_{m}(u) \prod_{i=1}^{m} \prod_{j=1}^{n+m+2}\left|u_{i}-t_{j}\right|^{-2-2 p_{j}} d^{2} \vec{u}_{m},
\end{aligned}
$$

где $\sum p_{k}=-n-1$.

Оно обобщает хорошо известное соотношение звезда-треугольник, которое отвечает случаю $m=0$ и $n=1$. Уравнение для асимптотики (1.25) ядра (1.21) легко получить последовательным применением этого соотношения. Это же соотношение помогает определить процедуру слияния в корреляционной функции (1.24).

Следующее тождество можно использовать для доказательства свойств симметрии ядра (1.23):

$$
\begin{aligned}
\int \mid t & -\left.\xi_{1}\right|^{2 p_{1}}\left|t-\xi_{2}\right|^{2 p_{2}}\left|t-\xi_{3}\right|^{2 p_{3}}\left|t-\xi_{4}\right|^{2 p_{4}} d^{2} t= \\
= & \prod_{k=1}^{4} \gamma\left(1+p_{k}\right)\left|\xi_{1}-\xi_{2}\right|^{2+2 p_{1}+2 p_{2}}\left|\xi_{3}-\xi_{4}\right|^{2+2 p_{3}+2 p_{4}} \times \\
& \times \int\left|s-\xi_{1}\right|^{-2-2 p_{2}}\left|s-\xi_{2}\right|^{-2-2 p_{1}}\left|s-\xi_{3}\right|^{-2-2 p_{4}}\left|s-\xi_{4}\right|^{-2-2 p_{3}} d^{2} s
\end{aligned}
$$

где $\sum p_{k}=-2$. Это тождество можно применить к любым двум парам точек $\xi_{j}$ (в (А.2) они выбраны как $\xi_{1}, \xi_{2}$ и $\left.\xi_{3}, \xi_{4}\right)$. Применяя его три раза, получаем соотношение (А.1) при $m=1$ и $n=1$. 
Свойство факторизации интеграла (1.24). Имеет место следующее свойство интеграла (1.24):

$$
\begin{aligned}
\int \prod_{k=1}^{n}\left|t_{k}\right|^{2 A}\left|t_{k}-1\right|^{2 B} K_{n}^{\Delta}\left(t_{1}, \ldots, t_{n} \mid z_{1}, \ldots, z_{n}\right) \mathcal{D}_{n}^{2 g}(t) d^{2} \vec{t}_{n}= \\
=\Lambda_{k}(A, B, \Delta) \prod_{j=n-k+1}^{n}\left|z_{j}\right|^{2(1+A+\Delta)}\left|z_{j}-1\right|^{2(1+B+\Delta)} \times \\
\quad \times \int \prod_{j=1}^{n-k}\left|t_{j}\right|^{2(A+k g)}\left|t_{j}-1\right|^{2(B+k g)} K_{n-k}^{\Delta-k g}\left(t_{1}, \ldots, t_{n-k} \mid z_{1}, \ldots, z_{n-k}\right) \times \\
\quad \times \mathcal{D}_{n-k}^{2 g}(t) d^{2} \vec{t}_{n-k} \prod_{i, j}\left|t_{i}-y_{j}\right|^{-2 g} \times \\
\quad \times \prod_{j=1}^{k}\left|y_{j}\right|^{-2(1+A+(k-1) g)}\left|y_{j}-1\right|^{-2(1+A+(k-1) g)} \times \\
\quad \times K_{k}^{-1-\Delta+(k-1) g}\left(y_{1}, \ldots, y_{k} \mid z_{n-k+1}, \ldots, z_{n}\right) \mathcal{D}_{k}^{2 g}(y) d^{2} \vec{y}_{k},
\end{aligned}
$$

где

$$
\Lambda_{k}(A, B, \Delta)=\prod_{j=0}^{k-1} \frac{\gamma((n-j) g)}{\gamma((j+1) g)} \frac{\gamma(1+A+j g) \gamma(1+B+j g) \gamma(1+\Delta-j g)}{\gamma(2+A+B+\Delta+(m-1-j) g)} .
$$

Это свойство позволяет существенно уменьшить число интегрирований в корреляционной функции

$$
\left\langle V_{-m_{1} b / 2}\left(z_{1}\right) \ldots V_{-m_{k} b / 2}\left(z_{k}\right) V_{\alpha_{1}}(0) V_{\alpha_{2}}(1) V_{\alpha_{3}}(\infty)\right\rangle .
$$

Предположим, что $m_{1} \geqslant m_{2} \geqslant \cdots \geqslant m_{k}$. Тогда, используя свойство факторизации (А.3) и соотношение (А.1), можно показать, что число интегрирований $N$ в корреляционной функции (А.4) можно уменьшить до

$$
\frac{N}{2}=\sum_{j=1}^{[k / 2]} j\left(m_{2 j}+m_{2 j-1}\right)+\frac{m_{k}(k+1)}{4}\left(1-(-1)^{k}\right) .
$$

В частности, при $m_{1}=m_{2}=\cdots=m_{k}=1$ число интегрирований (А.5) в корреляционной функции (А.4) равно

$$
N=\frac{k(k+2)}{2}+\frac{1}{4}\left(1-(-1)^{k}\right)
$$

Заметим, что число интегрирований в выражении (1.24) для этой корреляционной функции равно $k(k+1)$.

Аналитические свойства интеграла (1.16). Удобно рассмотреть более общий интеграл (интеграл (1.16) соответствует выбору $\xi_{1}=0, \xi_{2}=1, \xi_{3}=z, p_{1}=A, p_{2}=B$, $p_{3}=C$ и $\left.g=-b^{2}\right)$ :

$$
\mathbf{J}_{m}\left(p_{1}, p_{2}, p_{3} \mid \xi_{1}, \xi_{2}, \xi_{3}\right)=\int \prod_{k=1}^{m}\left|t_{k}-\xi_{1}\right|^{2 p_{1}}\left|t_{k}-\xi_{2}\right|^{2 p_{2}}\left|t_{k}-\xi_{3}\right|^{2 p_{3}} \mathcal{D}_{m}^{2 g}(t) d^{2} \vec{t}_{m}
$$


Этот интеграл удовлетворяет следующим замечательным соотношениям, которые можно использовать для аналитического продолжения $\mathbf{J}_{m}\left(p_{1}, p_{2}, p_{3} \mid \xi_{1}, \xi_{2}, \xi_{3}\right)$ как функции параметров $p_{1}, p_{2}$ и $p_{3}$. А именно, для любой пары точек $\xi_{j}$ (например, $\xi_{1}$ и $\left.\xi_{2}\right)$ имеем

$$
\begin{aligned}
& \mathbf{J}_{m}\left(p_{1}, p_{2}, p_{3} \mid \xi_{1}, \xi_{2}, \xi_{3}\right)=G_{m}\left(p_{1}, p_{2}, p_{3}\right)\left|\xi_{1}-\xi_{2}\right|^{2 m\left(1+p_{1}+p_{2}+(m-1) g\right)} \times \\
& \times \mathbf{J}_{m}\left(-1-p_{2}-(m-1) g,-1-p_{1}-(m-1) g,-1-p_{4}-(m-1) g \mid \xi_{1}, \xi_{2}, \xi_{3}\right),
\end{aligned}
$$

где

$$
G_{m}\left(p_{1}, p_{2}, p_{3}\right)=\prod_{j=0}^{m-1} \gamma\left(1+p_{1}+j g\right) \gamma\left(1+p_{2}+j g\right) \gamma\left(1+p_{3}+j g\right) \gamma\left(1+p_{4}+j g\right)
$$

и $p_{4}=-2-p_{1}-p_{2}-p_{3}-2(m-1) g$. Применяя это соотношение три раза, получаем

$$
\begin{aligned}
& \mathbf{J}_{m}\left(p_{1}, p_{2}, p_{3} \mid \xi_{1}, \xi_{2}, \xi_{3}\right)=G_{m}\left(p_{1}, p_{2}, p_{3}\right) \prod_{i<j}\left|\xi_{i}-\xi_{j}\right|^{2 m\left(1+p_{i}+p_{j}+(m-1) g\right)} \times \\
& \times \mathbf{J}_{m}\left(-1-p_{1}-(m-1) g,-1-p_{2}-(m-1) g,-1-p_{3}-(m-1) g \mid \xi_{1}, \xi_{2}, \xi_{3}\right) .
\end{aligned}
$$

Рассматривая аналитические свойства левой и правой частей в (А.6) и (А.7), можно заключить, что функция

$$
\tilde{\mathbf{J}}_{m}\left(p_{1}, p_{2}, p_{3} \mid \xi_{1}, \xi_{2}, \xi_{3}\right)=\prod_{k=1}^{4} \prod_{j=0}^{m-1} \Gamma^{-1}\left(1+p_{k}+j g\right) \mathbf{J}_{m}\left(p_{1}, p_{2}, p_{3} \mid \xi_{1}, \xi_{2}, \xi_{3}\right)
$$

является целой функцией параметров $p_{1}, p_{2}$ и $p_{3}$ для всех конечных и несовпадающих точек $\xi_{k}$. Подобные, но более сложные соотношения можно получить и для интеграла (1.19).

\section{ПРИЛОЖЕНИЕ Б}

\section{Вычисление ядра}

В данном приложении мы дадим некоторые указания по поводу доказательства формулы (1.24). Ход рассуждений тот же, что и при выводе трехточечной корреляционной функции в разделе 1 . Начнем с корреляционной функции

$$
\left\langle V_{-b / 2}\left(z_{1}\right) \ldots V_{-b / 2}\left(z_{m}\right) V_{\alpha_{1}}(0) V_{\alpha_{2}}(1) V_{\alpha_{3}}(\infty)\right\rangle
$$

и предположим, что выполнено условие экранирования, т.е.

$$
-\frac{m b}{2}+\alpha_{1}+\alpha_{2}+\alpha_{3}+n b=Q .
$$

С помощью (1.5) эта корреляционная функция выражается через $2 n$-мерный кулоновский интеграл

$$
\mathfrak{J}_{m}^{(n)}\left(A, B \mid z_{1}, \ldots, z_{m}\right)=\int \prod_{k=1}^{n}\left|t_{k}\right|^{2 A}\left|t_{k}-1\right|^{2 B} \prod_{j=1}^{m}\left|t_{k}-z_{j}\right|^{-2 g} \mathcal{D}_{n}^{2 g}(t) d^{2} \vec{t}_{n},
$$


где для краткости мы положили $A=-2 b \alpha_{1}, B=-2 b \alpha_{2}, g=-b^{2}$. Основная идея состоит в применении тождества (1.7) несколько раз и в сведении интеграла (Б.2) к другому интегралу с числом интегрирований, не зависящим от числа экранирующих полей $n$ (зависимость от $n$ появляется в этом интеграле как параметр). После этого можно продолжить данный интеграл на нецелые значения $n$, выражая $n$ из (Б.1).

Покажем, как такой подход работает для случаев четырех- и пятиточечных корреляционных функций. Далее в данном приложении мы многократно используем тождество (1.7), опуская для краткости несущественные множители, состоящие из $\gamma$-функций ${ }^{7)}$.

Четырехточечная функция. В случае четырехточечной функции имеем интеграл

$$
\mathfrak{J}_{1}^{(n)}(A, B \mid z)=\int \prod_{k=1}^{n}\left|t_{k}\right|^{2 A}\left|t_{k}-1\right|^{2 B}\left|t_{k}-z\right|^{-2 g} \mathcal{D}_{n}^{2 g}(t) d^{2} \vec{t}_{n} .
$$

Для начала используем тождество (1.7) справа налево и подставим

$$
\mathcal{D}_{n}^{2 g}(t) \sim \mathcal{D}_{n}(t) \int \mathcal{D}_{n-1}(y) \prod_{i=1}^{n-1} \prod_{j=1}^{n}\left|y_{i}-t_{j}\right|^{-2+2 g} d^{2} \vec{y}_{n-1}
$$

в (Б.3). Здесь и ниже знак “ " означает, что опущены несущественные числовые множители (ср. с (1.8), где такой множитель приведен в явном виде). После этого, снова используя тождество (1.7), можно проинтегрировать по переменным $t_{k}$ и преобразовать этот интеграл в двумерный интеграл по вспомогательной переменной $\xi$ :

$$
\begin{aligned}
& \int \prod_{k=1}^{n}\left|t_{k}\right|^{2 A}\left|t_{k}-1\right|^{2 B}\left|t_{k}-z\right|^{-2 g} \prod_{j=1}^{n-1}\left|t_{k}-y_{j}\right|^{-2+2 g} \mathcal{D}_{n}(t) d^{2} \vec{t}_{n} \sim \\
& \sim|z|^{2(1+A-g)}|z-1|^{2(1+B-g)} \prod_{k=1}^{n-1}\left|y_{k}\right|^{2(A+g)}\left|y_{k}-1\right|^{2(B+g)} \mathcal{D}_{n-1}^{2 g-1}(y) \times \\
& \quad \times \int|\xi|^{-2-2 A}|\xi-1|^{-2-2 B}|\xi-z|^{-2+2 g} \prod_{j=1}^{n-1}\left|\xi-y_{j}\right|^{-2 g} d^{2} \xi
\end{aligned}
$$

В результате получаем следующее соотношение между интегралами $\mathfrak{J}_{1}^{(n)}$ и $\mathfrak{J}_{1}^{(n-1)}$ :

$$
\begin{aligned}
& \mathfrak{J}_{1}^{(n)}(A, B \mid z) \sim|z|^{2(1+A-g)}|z-1|^{2(1+B-g)} \times \\
& \quad \times \int|\xi|^{-2(1+A)}|\xi-1|^{-2(1+B)}|\xi-z|^{-2+2 g} \mathfrak{J}_{1}^{(n-1)}(A+g, B+g \mid \xi) d^{2} \xi
\end{aligned}
$$

Снова применяя соотношение (Б.5), получаем

$$
\begin{aligned}
\mathfrak{J}_{1}^{(n)}(A, B \mid z) \sim|z|^{2(1+A-g)}|z-1|^{2(1+B-g)} \times \\
\quad \times \int|\xi-z|^{-2+2 g}|\nu|^{-2(1+A+g)}|\nu-1|^{-2(1+B+g)}|\nu-\xi|^{-2+2 g} \times \\
\quad \times \mathfrak{J}_{1}^{(n-2)}(A+2 g, B+2 g \mid \nu) d^{2} \xi d^{2} \nu .
\end{aligned}
$$

7)Эти множители легко восстановить в окончательном ответе исходя из общих принципов. 
Интеграл по $d^{2} \xi$ легко вычислить: с точностью до тривиального множителя он равен $|\nu-z|^{-2+4 g}$. В результате получаем следующее соотношение между интегралами $\mathfrak{J}_{1}^{(n)}$ и $\mathfrak{J}_{1}^{(n-2)}$ :

$$
\begin{aligned}
\mathfrak{J}_{1}^{(n)}(A, B \mid z) \sim|z|^{2(1+A-g)}|z-1|^{2(1+B-g)} \times \\
\quad \times \int|\nu|^{-2(1+A+g)}|\nu-1|^{-2(1+B+g)}|\nu-z|^{-2+4 g} \times \\
\quad \times \mathfrak{J}_{1}^{(n-2)}(A+2 g, B+2 g \mid \nu) d^{2} \nu .
\end{aligned}
$$

Вместо уравнений (Б.5) и (Б.6) после применения этой процедуры $k$ раз получаем:

$$
\begin{aligned}
\mathfrak{J}_{1}^{(n)}(A, B \mid z) \sim|z|^{2(1+A-g)}|z-1|^{2(1+B-g)} \times \\
\quad \times \int|\nu|^{-2(1+A+(k-1) g)}|\nu-1|^{-2(1+B+(k-1) g)}|\nu-z|^{-2+2 k g} \times \\
\quad \times \mathfrak{J}_{1}^{(n-k)}(A+k g, B+k g \mid \nu) d^{2} \nu .
\end{aligned}
$$

$\mathrm{B}$ частности, при $k=n$ имеем

$$
\begin{aligned}
\mathfrak{J}_{1}^{(n)}(A, B \mid z) & \sim|z|^{2(1+A-g)}|z-1|^{2(1+B-g)} \times \\
& \times \int|\nu|^{-2(1+A+(n-1) g)}|\nu-1|^{-2(1+B+(n-1) g)}|\nu-z|^{-2+2 n g} d^{2} \nu \sim \\
& \sim \int|\tau|^{2(A+(n-1) g)}|\tau-1|^{2(B+(n-1) g)}|\tau-z|^{-2 n g} d^{2} \tau
\end{aligned}
$$

где в последней строке мы преобразовали интеграл по переменной $\nu$, используя тождество (1.7). Интеграл (Б.7) можно переписать, используя определение ядра (1.22) в виде

$$
\mathfrak{J}_{1}^{(n)}(A, B \mid z) \sim \int|\tau|^{2(A+(n-1) g)}|\tau-1|^{2(B+(n-1) g)} K_{1}^{-n g}(\tau \mid z) d^{2} \tau .
$$

Полный множитель в (Б.8) можно получить, полагая $z=0$.

Пятиточечная функция. Рассмотрим теперь интеграл для пятиточечной функции

$$
\mathfrak{J}_{2}^{(n)}\left(A, B \mid z_{1}, z_{2}\right)=\int \prod_{k=1}^{n}\left|t_{k}\right|^{2 A}\left|t_{k}-1\right|^{2 B}\left|t_{k}-z_{1}\right|^{-2 g}\left|t_{k}-z_{2}\right|^{-2 g} \mathcal{D}_{n}^{2 g}(t) d^{2} \vec{t}_{n} .
$$

Подставляя $\mathcal{D}_{n}^{2 g}(t)$ в (Б.9) в виде (Б.4) и интегрируя по переменным $t_{k}$ с использованием (1.7), найдем следующее соотношение между интегралами $\mathfrak{J}_{2}^{(n)}$ и $\mathfrak{J}_{2}^{(n-1)}$ :

$$
\begin{aligned}
\mathfrak{J}_{2}^{(n)}\left(A, B \mid z_{1}, z_{2}\right) \sim & \prod_{k=1}^{2}\left|z_{k}\right|^{2(1+A-g)}\left|z_{k}-1\right|^{2(1+B-g)}\left|z_{1}-z_{2}\right|^{2-4 g} \times \\
& \times \int \prod_{j=1}^{2}\left|\xi_{j}\right|^{-2(1+A)}\left|\xi_{j}-1\right|^{-2(1+B)}\left|\xi_{j}-z_{1}\right|^{-2+2 g}\left|\xi_{j}-z_{2}\right|^{-2+2 g} \times
\end{aligned}
$$




$$
\times \mathfrak{J}_{2}^{(n-1)}\left(A+g, B+g \mid \xi_{1}, \xi_{2}\right) \mathcal{D}_{2}(\xi) d^{2} \vec{\xi}_{2} .
$$

Применяя соотношение (Б.10) дважды, получаем

$$
\begin{aligned}
& \mathfrak{J}_{2}^{(n)}\left(A, B \mid z_{1}, z_{2}\right) \sim \prod_{k=1}^{2}\left|z_{k}\right|^{2(1+A-g)}\left|z_{k}-1\right|^{2(1+B-g)}\left|z_{1}-z_{2}\right|^{2-4 g} \times \\
& \times \int \prod_{j=1}^{2}\left|\nu_{j}\right|^{-2(1+A+g)}\left|\nu_{j}-1\right|^{-2(1+B+g)} \times \\
& \times \mathfrak{J}_{2}^{(n-2)}\left(A+2 g, B+2 g \mid \nu_{1}, \nu_{2}\right) \mathcal{D}_{2}(\nu) d^{2} \vec{\nu}_{2} \times \\
& \times \prod_{k=1}^{2}\left|\xi_{k}-\nu_{1}\right|^{-2+2 g}\left|\xi_{k}-\nu_{2}\right|^{-2+2 g}\left|\xi_{k}-z_{1}\right|^{-2+2 g}\left|\xi_{k}-z_{2}\right|^{-2+2 g} \times \\
& \times \mathcal{D}_{2}^{2-2 g}(\xi) d^{2} \vec{\xi}_{2} .
\end{aligned}
$$

Интеграл по $d^{2} \vec{\xi}_{2}$ в формуле (Б.11) можно свести к двумерному интегралу, используя следующее тождество ${ }^{8)}$, которое можно вывести с помощью (1.7) и (А.1):

$$
\begin{aligned}
\int \prod_{k=1}^{2} \mid \xi_{k} & -\left.\nu_{1}\right|^{-2+2 g}\left|\xi_{k}-\nu_{2}\right|^{-2+2 g}\left|\xi_{k}-z_{1}\right|^{-2+2 g}\left|\xi_{k}-z_{2}\right|^{-2+2 g} \mathcal{D}_{2}^{2-2 g}(\xi) d^{2} \xi_{1} d^{2} \xi_{2}= \\
=2 & \frac{\gamma^{5}(g) \gamma(2-3 g)}{\gamma(2 g)}\left|\nu_{1}-\nu_{2}\right|^{4 g-2}\left|\nu_{1}-z_{1}\right|^{4 g-2}\left|\nu_{2}-z_{1}\right|^{4 g-2} \times \\
& \times \int\left|y-z_{1}\right|^{-2 g}\left|y-z_{2}\right|^{6 g-4}\left|y-\nu_{1}\right|^{-2 g}\left|y-\nu_{2}\right|^{-2 g} d^{2} y
\end{aligned}
$$

В результате применения тождества (Б.12) получаем

$$
\begin{aligned}
& \mathfrak{J}_{2}^{(n)}\left(A, B \mid z_{1}, z_{2}\right) \sim \prod_{k=1}^{2}\left|z_{k}\right|^{2(1+A-g)}\left|z_{k}-1\right|^{2(1+B-g)}\left|z_{1}-z_{2}\right|^{2-4 g} \times \\
& \times \int \prod_{j=1}^{2}\left|\nu_{j}\right|^{-2(1+A+g)}\left|\nu_{j}-1\right|^{-2(1+B+g)}\left|\nu_{j}-z_{1}\right|^{4 g-2} \times \\
& \times \mathfrak{J}_{2}^{(n-2)}\left(A+2 g, B+2 g \mid \nu_{1}, \nu_{2}\right) \mathcal{D}_{2}^{2 g}(\nu) d^{2} \vec{\nu}_{2} \times \\
& \times\left|y-z_{1}\right|^{-2 g}\left|y-z_{2}\right|^{6 g-4}\left|y-\nu_{1}\right|^{-2 g}\left|y-\nu_{2}\right|^{-2 g} d^{2} y .
\end{aligned}
$$

Равенство (Б.13) теперь “стабилизировано”. Это означает, что после применения соотношения (Б.10) можно выполнить вспомогательные интегрирования, а остающийся интеграл имеет аналогичный вид, и только параметры в подынтегральном выражении изменяются:

$$
\mathfrak{J}_{2}^{(n)}\left(A, B \mid z_{1}, z_{2}\right) \sim \prod_{k=1}^{2}\left|z_{k}\right|^{2(1+A-g)}\left|z_{k}-1\right|^{2(1+B-g)}\left|z_{1}-z_{2}\right|^{2-4 g} \times
$$

\footnotetext{
8)Это простое интегральное тождество отражает, в частности, интересный факт, что четырехточечная корреляционная функция полей $\Phi_{b / 2}$ в ОММ с центральным зарядом $c=1-6\left(b-b^{-1}\right)^{2}$ (интеграл в правой части (Б.12)) совпадает с точностью до тривиального множителя с четырехточечной корреляционной функцией полей $\Phi_{b^{\prime} / 2}$ в другой ОММ с центральным зарядом $c^{\prime}$, соответствующим параметру $b^{\prime 2}=1-b^{2}$ (интеграл в левой части (Б.12)).
} 


$$
\begin{aligned}
& \times \int \prod_{j=1}^{2}\left|\nu_{j}\right|^{-2(1+A+2 g)}\left|\nu_{j}-1\right|^{-2(1+B+2 g)}\left|\nu_{j}-z_{1}\right|^{6 g-2} \times \\
& \times \mathfrak{J}_{2}^{(n-3)}\left(A+3 g, B+3 g \mid \nu_{1}, \nu_{2}\right) \mathcal{D}_{2}^{2 g}(\nu) d^{2} \vec{\nu}_{2} \times \\
& \times\left|y-z_{1}\right|^{-4 g}\left|y-z_{2}\right|^{8 g-4}\left|y-\nu_{1}\right|^{-2 g}\left|y-\nu_{2}\right|^{-2 g} d^{2} y .
\end{aligned}
$$

Если повторить эту процедуру $n$ раз, получаем, что

$$
\begin{aligned}
& \mathfrak{J}_{2}^{(n)}(\left.A, B \mid z_{1}, z_{2}\right) \sim \prod_{k=1}^{2}\left|z_{k}\right|^{2(1+A-g)}\left|z_{k}-1\right|^{2(1+B-g)}\left|z_{1}-z_{2}\right|^{2-4 g} \times \\
& \quad \times \int \prod_{j=1}^{2}\left|\nu_{j}\right|^{-2(1+A+(n-1) g)}\left|\nu_{j}-1\right|^{-2(1+B+(n-1) g)}\left|\nu_{j}-z_{1}\right|^{2 n g-2} \mathcal{D}_{2}^{2 g}(\nu) d^{2} \vec{\nu}_{2} \times \\
& \quad \times\left|y-z_{1}\right|^{-2(n-1) g}\left|y-z_{2}\right|^{2(n+1) g-4}\left|y-\nu_{1}\right|^{-2 g}\left|y-\nu_{2}\right|^{-2 g} d^{2} y
\end{aligned}
$$

Используя тождества (1.7) и (А.2), преобразуем интеграл в правой части (Б.14) к виду

$$
\begin{aligned}
\mathfrak{J}_{2}^{(n)}\left(A, B \mid z_{1}, z_{2}\right) \sim \\
\sim \int \prod_{j=1}^{2}\left|\nu_{j}\right|^{2(A+(n-2) g)}\left|\nu_{j}-1\right|^{2(B+(n-2) g)}\left|\nu_{j}-z_{1}\right|^{-2(n-1) g} \mathcal{D}_{2}(\nu) d^{2} \vec{\nu}_{2} \times \\
\quad \times\left|y-z_{1}\right|^{2(n-2) g}\left|y-z_{2}\right|^{-2 n g}\left|y-\nu_{1}\right|^{-2+2 g}\left|y-\nu_{2}\right|^{-2+2 g} d^{2} y
\end{aligned}
$$

Соотношение (Б.15) можно выразить в терминах интеграла с ядром (1.23):

$$
\begin{aligned}
\mathfrak{J}_{2}^{(n)}\left(A, B \mid z_{1}, z_{2}\right) \sim \int & \prod_{j=1}^{2}\left|\nu_{j}\right|^{2(A+(n-2) g)}\left|\nu_{j}-1\right|^{2(B+(n-2) g)} \times \\
& \times K_{2}^{-(n-1) g}\left(\nu_{1}, \nu_{2} \mid z_{1}, z_{2}\right) \mathcal{D}_{2}^{2 g}(\nu) d^{2} \vec{\nu}_{2} .
\end{aligned}
$$

$(m+3)$-Точечная функция. Для общих $m$ интеграл (Б.2) можно упростить таким же образом. Можно обобщить интегральные соотношения (Б.5) и (Б.10):

$$
\begin{aligned}
\mathfrak{J}_{m}^{(n)}\left(A, B \mid z_{1}, \ldots, z_{m}\right) \sim & \prod_{k=1}^{m}\left|z_{k}\right|^{2(1+A-g)}\left|z_{k}-1\right|^{2(1+B-g)} \prod_{i<j}\left|z_{i}-z_{j}\right|^{2-4 g} \times \\
& \times \int \prod_{j=1}^{m}\left|\xi_{j}\right|^{-2(1+A)}\left|\xi_{j}-1\right|^{-2(1+B)} \prod_{k=1}^{m}\left|\xi_{j}-z_{k}\right|^{-2+2 g} \times \\
& \times \mathfrak{J}_{m}^{(n-1)}\left(A+g, B+g \mid \xi_{1}, \ldots, \xi_{m}\right) \mathcal{D}_{m}(\xi) d^{2} \vec{\xi}_{m} .
\end{aligned}
$$

Применяя это соотношение $m$ раз, можно показать, что интеграл стабилизируется ${ }^{9)}$. Это означает, что после следующих применений соотношения (Б.17) он имеет тот

\footnotetext{
9) Для этого следует использовать тождество, которое обобщает (Б.12) для большего числа "внешних" точек $z_{i}$ и $\nu_{j}$.
} 
же вид (со сдвинутыми параметрами $A$ и $B$ ), но с меньшим числом интегрирований. Окончательная формула выглядит аналогично (Б.8) и (Б.16):

$$
\begin{aligned}
& \mathfrak{J}_{m}^{(n)}\left(A, B \mid z_{1}, \ldots, z_{m}\right) \sim \\
& \sim \int \prod_{j=1}^{m}\left|\nu_{j}\right|^{2(A+(n-m) g)}\left|\nu_{j}-1\right|^{2(B+(n-m) g)} \times \\
& \quad \times K_{m}^{-(n-m+1) g}\left(\nu_{1}, \ldots, \nu_{m} \mid z_{1}, \ldots, z_{m}\right) \mathcal{D}_{m}^{2 g}(\nu) d^{2} \vec{\nu}_{m}
\end{aligned}
$$

где $K_{m}^{\Delta}\left(\nu_{1}, \ldots, \nu_{m} \mid z_{1}, \ldots, z_{m}\right)$ имеет вид (1.21).

Благодарности. Работа В. Фатеева была поддержана European Committee в рамках контракта EUCLID HRPN-CT-2002-00325. Работа А. Литвинова была выполнена при поддержке РФФИ (грант № 07-02-00799-а), Программы поддержки ведущих научных школ (грант № НШ-2044.2003.2) и программы РАН “Элементарные частицы и фундаментальная ядерная физика". Важная часть работы была выполнена во время визита А. Литвинова в Laboratoire de Physique Théorique et Astroparticules Université Montpellier 2 в рамках программы ENS-LANDAU.

\section{Список литературы}

[1] A. M. Polyakov, Phys. Lett. B, 103:3 (1981), 207-210.

[2] V.S. Dotsenko, Modern Phys. Lett. A, 6:39 (1991), 3601-3612.

[3] P. Di Francesco, D. Kutasov, Nucl. Phys. B, 375:1 (1992), 119-170; arXiv: hep-th/9109005.

[4] А.Б. Замолодчиков, ТМФ, 142:2 (2005), 218-134; arXiv: hep-th/0505063.

[5] A. A. Belavin, A. B. Zamolodchikov, Писъма в ЖЭЭТФ, 82:1 (2005), 8-14.

[6] А. А. Белавин, А. Б. Замолодчиков, ТМФ, 147:3 (2006), 339-371.

[7] И. К. Костова, В. Б. Петкова, ТМФ, 146:1 (2006), 132-145; arXiv: hep-th/0505078.

[8] I. K. Kostov, V. B. Petkova, Nucl. Phys. B, 770:3 (2007), 273-331; arXiv: hep-th/0512346; Nucl. Phys. B, 769:3 (2007), 175-216; arXiv: hep-th/0609020.

[9] A. A. Belavin, A. M. Polyakov, A. B. Zamolodchikov, Nucl. Phys. B, 241:2 (1984), 333-380.

[10] V.S. Dotsenko, V. A. Fateev, Nucl. Phys. B, 240:3 (1984), 312-348.

[11] V.S. Dotsenko, V. A. Fateev, Nucl. Phys. B, 251:5-6 (1985), 691-734.

[12] M. Goulian, M. Li, Phys. Rev. Lett., 66:16 (1991), 2051-2055.

[13] P. Baseilhac, V. A. Fateev, Nucl. Phys. B, 532:3 (1998), 567-587; arXiv: hep-th/9906010.

[14] H. Dorn, H. J. Otto, Phys. Lett. B, 291:1-2 (1992), 39-43; arXiv: hep-th/9206053.

[15] H. Dorn, H. J. Otto, Nucl. Phys. B, 429:2 (1994), 375-388; arXiv: hep-th/9403141.

[16] A. B. Zamolodchikov, A. B. Zamolodchikov, Nucl. Phys. B, 477:2 (1996), 577-605; arXiv: hep-th/9506136.

[17] V.A. Fateev, A. V. Litvinov, Писъма в ЖКЭТФ, 84:10 (2006), 625-630.

[18] V.A. Fateev, A. V. Litvinov, Писъма в ЖКЭТФ, 81:11 (2005), 728-732; arXiv: hep-th/0505120. 